\title{
Algebraic Multiuser Space-Frequency Block Codes
}

\author{
Yi Hong \\ Institute of Advanced Telecom. \\ University of Wales, Swansea \\ y.hong@swansea.ac.uk
}

\author{
Emanuele Viterbo \\ DEIS - Università della Calabria \\ via P. Bucci 42C, Rende (CS), Italy \\ viterbo@deis.unical.it
}

\begin{abstract}
In this paper, we consider a wireless multiuser multiple-input multiple-output orthogonal frequency division multiplexing (MIMO-OFDM) uplink scenario, where the information sequences of all users are encoded by individual spacefrequency block codes (SFBC). At the receiver, joint maximum likelihood detection is applied using the sphere decoding algorithm. Using a truncated union-bound approximation, we propose the design criteria of multiuser SFBCs for frequency selective fading MIMO multiple access channels (MAC). Next, we show how, by combining the structure of algebraic perfect space-time block codes in [4], a family of multiuser SFBCs can be constructed to fulfill the design criteria. Finally, we show that the proposed SFBC outperforms previously known codes.
\end{abstract}

Index Terms-space-frequency codes, multiuser, MIMO, OFDM.

\section{INTRODUCTION}

Space-frequency codes (SFCs) have been intensively studied in $[1,2]$ for single-user multiple-input multiple-output (MIMO) orthogonal frequency division multiplexing (OFDM) systems over frequency selective fading channels. Recently, Gärtner and Bölcskei extended the idea of single user SFC to multiuser MIMO-OFDM over frequency selective fading MIMO multiple access channels (MACs) in [3]. With the aim of increasing information rate, in [3], the design criteria of multiuser space-frequency block codes (SFBCs) were proposed using a concept of dominant error regions. A $2 \times 2$ MIMO multiuser SFBC was proposed based on Alamouti structure.

In [6], authors generalized the multiuser SFBC design criteria for more than two users over a frequency selective fading MIMO MAC, based on minimizing an upper bound of pairwise error probability (PEP). The SFBCs were designed using a constellation rotation followed by a phase rotation, in order to enhance the multiuser diversity order [6]. However, these codes incur in large peak-to-average penalties, since some elements in the codeword matrices are zero.

In [10], an algebraic construction of multiuser SFBCs was recently proposed to achieve the diversity-multiplexing tradeoff [8] for users using a single transmit antenna $\left(n_{t}=1\right)$ and any number of receive antennas $\left(n_{r}\right)$.

In our paper, we consider the two transmit antenna case, i.e., $n_{t}=2$, which was also discussed in $[3,6]$. Unlike the multiuser codes in $[3,6]$, we propose the code design criterion over frequency selective fading MIMO MACs based on a truncated union-bound (UB) approximation. Motivated by algebraic perfect space-time block codes in [4], we show how to construct a family of multiuser SFBCs in order to minimize the error probability of the truncated UB, without the peak-toaverage penalty of [6]. Within this family, we present a code design example for a two-user $2 \times 2$ MIMO. Note that with QAM signalling, the MLD can be obtained using the sphere decoding (SD) algorithm. Next, we show that the proposed codes outperform the previously known SFBCs $[3,6]$.

Notations: Boldface letters are used for column vectors, and capital boldface letters for matrices. Superscripts ${ }^{T}$ and ${ }^{\dagger}$ denote transposition and Hermitian transposition, respectively. Let $\mathbb{C}$ denote the field of complex numbers.

The operator $\operatorname{diag}(\cdot, \ldots, \cdot)$ generates a block diagonal matrix with its arguments on diagonal. The $\operatorname{vec}(\cdot)$ operator stacks the $m$ column vectors of a $n \times m$ complex matrix into a $m n$ complex column vector. Let $\|\cdot\|$ denote the Frobenius norm and let $\mathbb{E}[\cdot]$ denote mean of a random variable.

Given a complex number $x$ we define the $(\tilde{)})$ operator from $\mathbb{C}$ to $\mathbb{R}^{2}$ as

$$
\tilde{x} \triangleq[\Re(x), \Im(x)]^{T}
$$

where $\Re(\cdot)$ and $\Im(\cdot)$ denote real and imaginary parts. The $(\tilde{)})$ operator can be extended to complex vectors $\mathbf{x}=$ $\left[x_{1}, \ldots x_{n}\right]^{T} \in \mathbb{C}^{n}$ as

$$
\tilde{\mathbf{x}} \triangleq\left[\Re\left(x_{1}\right), \Im\left(x_{1}\right), \ldots, \Re\left(x_{n}\right), \Im\left(x_{n}\right)\right]^{T}
$$

Given a complex number $x$, the $(\cdot)$ operator from $\mathbb{C}$ to $\mathbb{R}^{2 \times 2}$ is defined by

$$
\check{x} \triangleq\left[\begin{array}{cc}
\Re(x) & -\Im(x) \\
\Im(x) & \Re(x)
\end{array}\right]
$$

The (.) operator can be similarly extended to $n \times n$ matrices by applying it to all the entries, yielding $2 n \times 2 n$ real matrices. The following relation holds:

$$
\widetilde{\mathbf{A x}}=\check{\mathbf{A}} \tilde{\mathbf{x}}
$$

\section{SySTEM MODEL}

We consider an uplink scenario, where $K$ uncoordinated users simultaneously communicate with a base station over a frequency selective fading MIMO MAC. We assume that each user employs an identical MIMO-OFDM system with $n_{t}$ transmit antennas. We consider that the information sequences 
of all users are encoded by their individual SFBCs. At the BS, the receiver is assumed to have $n_{r}=n_{t}$ receive antennas.

\section{A. Transmitter}

Let $N_{F F T}$ be the number of total subcarriers in OFDM of each user. We assume that $N_{F F T}$ and $N$ are dividable and $N<N_{F F T}$. Therefore at the transmitter of each user, every $N$ information symbols can be fed into an SFBC encoder. The detailed encoding procedure is given as follows.

For each user $k$, an information vector of length $N$ is defined as

$$
\mathbf{s}^{(k)} \triangleq\left[s_{1}^{(k)}, \ldots, s_{i}^{(k)}, \ldots, s_{N}^{(k)}\right]^{T} \in \mathbb{C}^{N}
$$

where $\left\{s_{i}^{(k)}\right\}, i=1, \ldots, N$, are independent information symbols drawn from a complex $Q-\mathrm{QAM}$ constellation. The joint symbol vector of all $K$ users can be written as

$$
\mathbf{s}_{\text {joint }} \triangleq\left[\left(\mathbf{s}^{(1)}\right)^{T}, \ldots,\left(\mathbf{s}^{(k)}\right)^{T}, \ldots,\left(\mathbf{s}^{(K)}\right)^{T}\right]^{T} \in \mathbb{C}^{K N}
$$

The symbol vector of each user $\mathbf{s}^{(k)}$ is encoded by its individual SFBC yielding a SFBC-OFDM codeword matrix $\mathrm{C}_{k} \in \mathbb{C}^{n_{t} \times N}$ from the codebook $\mathcal{C}_{k}$, given by

$$
\mathbf{C}_{k} \triangleq\left[\left(\mathbf{c}_{1}^{(k)}\right)^{T}, \ldots,\left(\mathbf{c}_{j}^{(k)}\right)^{T}, \ldots,\left(\mathbf{c}_{n_{t}}^{(k)}\right)^{T}\right]^{T} \in \mathcal{C}_{k}
$$

with $k=1, \ldots, K$. The vectors $\mathbf{c}_{j}^{(k)} \triangleq\left\{c_{j, n}^{(k)}\right\} \in \mathbb{C}^{1 \times N}$ for $j=1, \ldots, n_{t}$, denote the SFBC-OFDM symbol vector from the $j$-th transmit antenna of user $k$.

We also assume that all $K$ users simultaneously transmit their individual SFBC-OFDM symbol matrices over $N$ subcarriers, yielding the following joint codeword matrix:

$$
\mathbf{X} \triangleq\left[\mathbf{C}_{1}^{T}, \ldots, \mathbf{C}_{k}^{T}, \ldots, \mathbf{C}_{K}^{T}\right]^{T} \in \mathcal{C}
$$

where $\mathcal{C}$ is the joint codebook.

In this paper, we assume that each user employs a linear SFBC [5, Definition 5], so that the elements $c_{j, n}^{(k)}$, for all $j$ and $n$, are linear combinations of $N$ complex $Q-\mathrm{QAM}$ symbols and are transmitted from the $j$-th transmit antenna over the $n$-th subcarrier. Then we have the following relation:

$$
\widetilde{\operatorname{vec}(\mathbf{X})}=\mathbb{G} \tilde{\mathbf{s}}_{\text {joint }}
$$

where $\mathbb{G} \in \mathbb{R}^{2 n_{t} K N \times 2 K N}$ is called the (real) generator matrix of the linear code [5, Definition 5]. This relation is particularly useful in the following to describe the sphere decoding of the multiuser MIMO-OFDM.

\section{B. Receiver}

At the receiver, after matched filtering, sampling and fast Fourier transform (FFT), the received signal vector $\mathbf{y} \in$ $\mathbb{C}^{K N n_{r}}$ in frequency domain can be written as

$$
\mathbf{y}=\mathcal{H} \operatorname{vec}(\mathbf{X})+\mathbf{n},
$$

where

1) $\mathbf{y} \triangleq\left[\mathbf{y}(1)^{T}, \ldots, \mathbf{y}(n)^{T}, \ldots, \mathbf{y}(N)^{T}\right]^{T}$, the vector $\mathbf{y}(n)=\left[y_{1}(n), \ldots, y_{n_{r}}(n)\right]^{T}$ represents the received signal vector from all $K$ users over the $n$-th subcarrier; in particular, the element $y_{i}(n), i=1, \ldots, n_{r}$, denotes the superposition of the received signals from all $K$ users at the $i$-th receive antenna on the $n$-th subcarrier.

2) $\mathbf{n} \in \mathbb{C}^{N n_{r}}$ is the complex white Gaussian noise with i.i.d. samples $\sim \mathcal{N}_{\mathbb{C}}\left(0, N_{0}\right)$.

3) $\mathcal{H} \in \mathbb{C}^{N n_{r} \times K N n_{t}}$ is defined as

$$
\mathcal{H} \triangleq \operatorname{diag}\{\mathbf{H}(1), \ldots, \mathbf{H}(n), \ldots, \mathbf{H}(N)\}
$$

with

$$
\mathbf{H}(n)=\left[\mathbf{H}^{(1)}(n), \ldots, \mathbf{H}^{(k)}(n), \ldots, \mathbf{H}^{(K)}(n)\right]
$$

where $\mathbf{H}^{(k)}(n) \triangleq\left\{H_{i, j}^{(k)}(n)\right\} \in \mathbb{C}^{n_{r} \times n_{t}}, k=1, \ldots, K$, $i=1, \ldots, n_{r}, j=1, \ldots, n_{t}, n=1, \ldots, N$, denotes the channel matrix in frequency domain associated with the $k$-th user over the $n$-th subcarrier. The elements $H_{i, j}^{(k)}(n)$ are the channel frequency response from the $j$ th transmit antenna to the $i$-th receive antenna over the $n$-th subcarrier for user $k$. Also, we assume that each element $H_{i, j}^{(k)}(n)$ is i.i.d. circularly symmetric Gaussian random variable $\sim \mathcal{N}_{\mathbb{C}}(0,1)$.

In this paper, we consider a frequency selective fading MIMO MAC, i.e., the channel coefficients $H_{i, j}^{(k)}(n)$ are assumed to be constant for $N$ subcarriers, and vary independently from one codeword to the next. Note that this could model the case of a OFDM system with $N_{F F T}>N$ subcarriers, where $N$ adjacent subcarriers span the channel coherence bandwidth.

\section{Sphere Decoding}

Separating real and imaginary parts in (3), we obtain

$$
\begin{aligned}
& \tilde{\mathbf{y}}=\check{\mathcal{H}} \widehat{\operatorname{vec}(\mathbf{X})}+\tilde{\mathbf{n}} \\
& =\check{\mathcal{H}} \mathbb{G} \tilde{\mathbf{S}}_{\text {joint }}+\tilde{\mathbf{n}}
\end{aligned}
$$

In order to simplify the notation let

$$
\begin{aligned}
\Theta & =\check{\mathcal{H}} \times \mathbb{G} \\
\mathbf{r} & =\left[r_{1}, \ldots, r_{2 K N n_{r}}\right]^{T \triangleq \tilde{\mathbf{y}}} \\
\mathbf{u} & =\left[u_{1}, \ldots, u_{2 K N}\right]^{T} \triangleq \tilde{\mathbf{s}}_{\text {joint }} \\
\mathbf{w} & =\left[w_{1}, \ldots, w_{2 K N n_{r}}\right]^{T \triangleq \tilde{\mathbf{n}}}
\end{aligned}
$$

so that we rewrite (4) as

$$
\mathbf{r}=\Theta \mathbf{u}+\mathbf{w}
$$

Thanks to the linearity of the code we can apply SD to perform the MLD. Assuming $u_{i} \in \mathcal{X}$, where $\mathcal{X}$ is a $|\mathcal{X}|$-PAM constellation, such that $\mathcal{X}^{2}=Q-\mathrm{QAM}$. Lattice decoding finds

$$
\hat{\mathbf{u}}=\arg \min _{\mathbf{u} \in \mathcal{X}^{2 K N}}\|\mathbf{r}-\Theta \mathbf{u}\|^{2}
$$

where $\hat{\mathbf{u}}=\left\{\hat{u}_{i}\right\}$ with $i=1, \ldots, 2 K N, \hat{u}_{i} \in \mathcal{X}$, and $\mathcal{X}^{2 K N}$ is the finite constellation carved from a $2 K N$-dimensional lattice with generator matrix $\Theta$. 


\section{New Multiuser Space-Frequency Block Codes}

In this Section, we present 1) the design criteria of multiuser SFBC over a frequency selective fading MIMO MAC; and 2) a design example of a two-user $2 \times 2$ MIMO-OFDMs with $N=4$. Finally, we compare the performance of the proposed code and previously known codes $[3,6]$.

\section{A. Multiuser SFBC Design Criteria (Full-Rank Design)}

For all $K$ users, assuming that a joint codeword matrix $\mathbf{X} \in \mathcal{C}$ is transmitted, it may occur that $\|\mathbf{Y}-\mathcal{H} \operatorname{vec}(\mathbf{X})\|^{2}>$ $\|\mathbf{Y}-\mathcal{H} \operatorname{vec}(\widehat{\mathbf{X}})\|^{2}$, with $\mathbf{X} \neq \widehat{\mathbf{X}}$, resulting in a pairwise error. Let $\mathbf{X}-\widehat{\mathbf{X}}$, with $\mathbf{X} \neq \widehat{\mathbf{X}}$, be the joint codeword-difference matrix and let $\mathbf{A} \triangleq(\mathbf{X}-\widehat{\mathbf{X}})(\mathbf{X}-\widehat{\mathbf{X}})^{\dagger}$ be the joint codeworddistance matrix.

Similarly, for the $k$-th user, assuming that codeword matrix $\mathbf{C}_{k} \in \mathcal{C}_{k}$ is transmitted and $\widehat{\mathbf{C}}_{k}$ is detected erroneously at the receiver, we call $\mathbf{C}_{k}-\widehat{\mathbf{C}}_{k}$ the user codeword difference matrix. The corresponding user codeword distance matrix is defined as $\mathbf{E}^{(k)} \triangleq\left(\mathbf{C}_{k}-\widehat{\mathbf{C}}_{k}\right)\left(\mathbf{C}_{k}-\widehat{\mathbf{C}}_{k}\right)^{\dagger}$. Let $r_{k}$ denote the minimum rank of $\mathbf{E}^{(k)}$ for all user codeword pairs in $\mathcal{C}_{k}$. We will assume $r_{k}=\min \left(n_{t}, N\right)$ for all $k$, i.e., all user codes have the same full-rank $r_{k}=r$.

If this full-rank condition holds for all $K$ users, it is not guaranteed that the joint codebook is also full rank. We will show in the following how to design the user codes in order to get a "full-rank" joint multiuser SFBC, defined such that if all $\mathbf{E}^{(k)} \neq \mathbf{0}$ then $\operatorname{rank}(\mathbf{A})=\min \left(K n_{t}, N\right)=K r$.

Here, we assume that there are $K$ users each with $n_{t}=2$ antennas, a receiver with $n_{r}=2$ antennas and $N=2 K$ subcarriers. Given the $k$-th user transmitted QAM information symbol vector $\mathbf{s}^{(k)}$, defined in (1), we use an algebraic unitary matrix $\mathbf{M}$ with full diversity ([9], [7]) to generate the coded symbol vector

$$
\mathbf{v}^{(k)}=\mathbf{M s}^{(k)}=\left[v_{1}^{(k)}, \ldots, v_{N}^{(k)}\right]^{T} \quad k=1, \ldots, N
$$

The matrix $\mathbf{M}$ is obtained from the canonical embedding of an integral basis $\left\{\omega_{j}\right\}, j=1, \ldots, N$ of an ideal of an algebraic number field $L$ of degree $N$ over $\mathbb{Q}(i)$ [8]. The full diversity property implies that all the elements of $\mathbf{v}^{(k)}$ are non-zero for any non-zero information vector $\mathbf{s}^{(k)}$ [8]. The user codewords are then generated as

$$
\begin{aligned}
C_{1} & =\left[\begin{array}{ccccc}
v_{1}^{(1)} & v_{2}^{(1)} & \ldots & & v_{N}^{(1)} \\
\gamma v_{N}^{(1)} & v_{1}^{(1)} & & \ldots & v_{N-1}^{(1)}
\end{array}\right] \\
C_{2} & =\left[\begin{array}{lllll}
\gamma v_{N-1}^{(2)} & \gamma v_{N}^{(2)} & \ldots & & v_{N-2}^{(2)} \\
\gamma v_{N-2}^{(2)} & \gamma v_{N-1}^{(2)} & \gamma v_{N}^{(2)} & \ldots & v_{N-3}^{(k)}
\end{array}\right] \\
C_{3} & =
\end{aligned}
$$

where $\gamma \neq 1$ is a complex number on the unit circle in order to preserve a uniform transmitted power from each antenna. In such a manner, the code will not incur in extra peak-toaverage penalty, since all entries are non-zero with the same average power.

Lemma 1: For $\gamma \neq 1$, the above user codes $\mathcal{C}_{k}$ are full rank $r=2$ for all $k=1, \ldots, K$ users.
Proof. It is enough to show that the two rows of $C_{k}$ are linearly independent, which is equivalent to saying they can not be scalar multiples for any non zero information vector $\mathbf{s}^{(k)}$. This is clearly the case thanks to the term $\gamma \neq 1$ which multiplies a different number of elements in each row.

Lemma 2: If $N=n_{t} K$ the joint codeword matrices $\mathbf{X}$ defined in (2) are square and the joint multiuser code $\mathcal{C}$ is "full-rank" if $\gamma$ is transcendental.

Proof. Looking at the structure of the $N \times N$ square codeword matrix $\mathbf{X}$ we note that the elements of the lower triangular part are multiplied by $\gamma$. It can be easily verified that the determinant of $\mathbf{X}$ is a polynomial $p(\gamma)$ in the variable $\gamma$ by using the well known expression

$$
\operatorname{det}(\mathbf{X})=\sum_{\pi \in S_{N}} \prod_{i=1}^{N} x_{i, \pi(i)}
$$

where the sum runs over all the permutations $\pi$ in the symmetric group $S_{N}$. This polynomial has degree $N-1$ since the coefficient of the term $\gamma^{N-1}$ is given by $x_{1, N}$. $x_{2,1} \cdot x_{3,2} \cdots x_{N, N-1} \neq 0$, which is not zero thanks to the full diversity rotation in (8), that yields vectors $\mathbf{v}^{(k)}$ with all non-zero entries. The coefficients of $p(\gamma)$ are in the algebraic number field $L$ defined after equation (8). The roots of the polynomial equation $p(\gamma)=0$ are in some algebraic extension $L^{\prime}$ of $L$ [8]. By choosing $\gamma$ to be transcendental (i.e. in no finite extension of $L$ ) we can guarantee that the $p(\gamma)=\operatorname{det}(\mathbf{X}) \neq 0$.

Note that the above Lemma gives only a necessary condition and some specific not transcendental $\gamma \mathrm{s}$ not belonging to $L^{\prime}$ can also yield a "full-rank" joint multiuser code.

\section{B. Multiuser SFBC Design Criteria}

To simplify analysis, we assume that the full-rank joint multiuser SFBC is linear [5]. Then, the error probability of the multiuser MIMO-OFDM is upper bounded by the following union bound [6]:

$$
P(e) \leq \sum_{\mathbf{X} \neq \mathbf{0}} \sum_{k=1}^{K} \sum_{\left(i_{1}, \ldots, i_{k}\right)}^{A_{k}} P\left(e_{i_{1}} \cap \cdots \cap e_{i_{k}} \mid \mathbf{X}\right)
$$

where $e_{k}$ represents the $k$-th user error event, the sum $\sum_{\left(i_{1}, \ldots, i_{k}\right)}^{A_{k}}$ is over all $A_{k} \triangleq\left(\begin{array}{c}K \\ k\end{array}\right)$ possible $k$-tuples of users in error. The $k$-tuple $\left(i_{1}, \ldots, i_{k}\right)$ denotes the indices of $k$ distinct users. Using the Chernoff bound, we then upper bound each term in (9) with:

$$
P\left(e_{i_{1}} \cap \cdots \cap e_{i_{k}} \mid \mathbf{X}\right) \leq\left(\frac{E_{s}}{N_{0}}\right)^{-n_{r} k r}\left[\delta_{\left(i_{1}, \ldots, i_{k}\right)}(\mathbf{X})\right]^{-n_{r}}
$$

where $E_{s}$ is the average energy per QAM information symbol and the determinants:

$$
\delta_{\left(i_{1}, \ldots, i_{k}\right)}(\mathbf{X}) \triangleq \operatorname{det}\left(\sum_{\ell=1}^{k} \mathbf{C}_{i_{\ell}} \mathbf{C}_{i_{\ell}}^{\dagger}\right)
$$




\begin{tabular}{|c|c|c|c|c|c|}
\hline Codes & $\delta_{1}^{(\min )}$ & $A_{1} B_{1}$ & $\delta_{2}^{(\min )}$ & $A_{2} B_{2}$ & SNR@10-3 \\
\hline \hline New & 13.2 & 16 & 52 & 16 & 13.8 \\
\hline GB & 16 & 64 & 32 & 256 & 14 \\
\hline ZL & 4 & 64 & 8 & 256 & 13.8 \\
\hline
\end{tabular}

TABLE I

COMPARISON OF MINIMUM DETERMINANTS WHEN ONE OR TWO USERS ARE IN ERROR, ASSOCIATED MULTIPLICITIES.

We can further define the corresponding minimum determinants among all the $k$-tuples

$$
\delta_{k}^{(\min )}=\min _{\substack{\left(i_{1}, \ldots, i_{k}\right) \\ \mathbf{X} \neq \mathbf{0}}} \delta_{\left(i_{1}, \ldots, i_{k}\right)}(\mathbf{X})
$$

Finally, we consider a truncated union bound based only on the terms corresponding to minimum determinants $\delta_{k}^{(\min )}$

$$
P(e) \approx \sum_{k=1}^{K} A_{k} B_{k} P\left(\delta_{k}^{(\mathrm{min})}\right)
$$

where the $A_{k} B_{k}$ is the multiplicity of the term

$$
P\left(\delta_{k}^{(\min )}\right)=\left(\frac{E_{s}}{N_{0}}\right)^{-n_{r} k r}\left(\delta_{k}^{(\min )}\right)^{-n_{r}}
$$

which represents the dominant error probability of a $k$-tuple of users. In particular, $B_{k}$ denotes the associate multiplicity of (12) for a given $A_{k}$.

The codes design in the previous section satisfy

Lemma 3: The determinants in (11) are all non-zero Proof. Since the terms $\mathbf{C}_{i_{\ell}} \mathbf{C}_{i_{\ell}}^{\dagger}$ in (11) are positive definite we use the determinant inequality

$$
\operatorname{det}\left(\sum_{\ell=1}^{k} \mathbf{C}_{i_{\ell}} \mathbf{C}_{i_{\ell}}^{\dagger}\right) \geq \sum_{\ell=1}^{k} \operatorname{det}\left(\mathbf{C}_{i_{\ell}} \mathbf{C}_{i_{\ell}}^{\dagger}\right)
$$

where the determinats on the rhs are all greater than zero due to Lemma 1.

Hence, under the full-rank and linearity assumption, in order to minimize the error probability $P(e)$, we should design the multiuser SFBCs to

1) maximize the minimum determinants $\delta_{k}^{(\min )}, \forall k$;

2) minimize the associated multiplicity $A_{k} B_{k}$.

\section{Example of new multiuser SFBC for frequency selective} fading MIMO MACs

As an example, we consider $K=2$ users each employing a $2 \times 2$ MIMO-OFDM with $N=4$ subcarriers. The unitary matrix $\mathbf{M}$ in [4] is chosen and $\gamma=i$. Note that this $\gamma$ is not transcendental but also guarantees the non-zero determinant. We also note that the proposed code and the known codes in $[3,6]$ are "full-rank" joint multiuser SFBCs, i.e., $r_{k}=2$ and $\operatorname{rank}(\mathbf{A})=4$. We recall that the error probability $P(e)$ takes into account the total number of errors of both users. Let us define the peak-signal-to-noise ratio as Peak-SNR $\triangleq n_{t} E_{p} / N_{0}$ where $E_{p}=\max _{i, j} \mathbb{E}\left[\left|x_{i, j}\right|^{2}\right]$ denotes the peak average energy

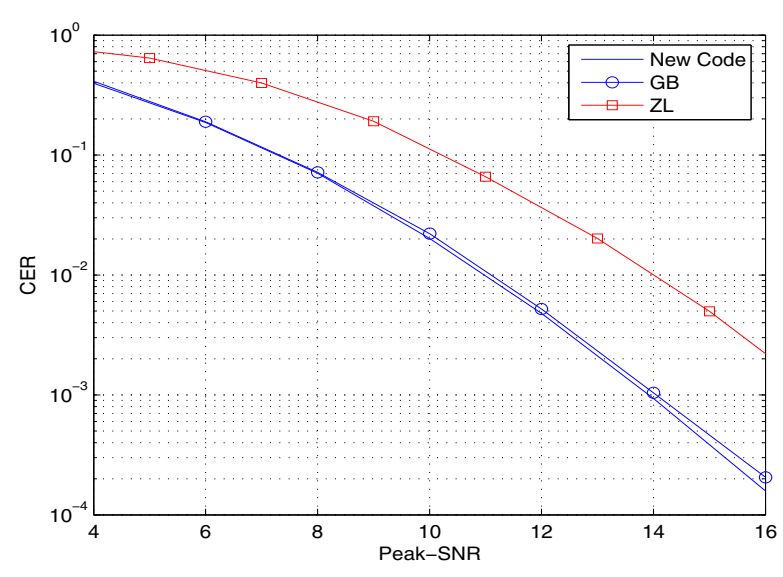

Fig. 1. Comparison of the CER performance of the new code, known codes in [3] and [6], 4-QAM signalling, frequency selective fading channel.

of a transmitted QAM symbol from one antenna. We have $E_{p}=E_{s}$ for the proposed code and the one in [3], while $E_{p}=2 E_{s}$ for the code in [6] which has some zero entries in the codeword.

We compare the proposed code with the multiuser SFBCs given in [3] and [6], for frequency selective fading MIMO MACs. In Table I we show the minimum determinant $\delta_{k}^{(\min )}$ when $k$ users are simultaneously in error, the associated multiplicities $A_{k} B_{k}$ and the SNR (dB) at codeword error rate (CER) of $10^{-3}$. In the table and in the following, we use the standard convention of denoting the codes by the initials of the authors who proposed them.

From Table I we see that: 1) when one user is in error, the minimum determinant of the code of [3] is slightly larger than that of the proposed code; 2) when both users are in error, the minimum determinants of our code is the largest among all multiuser SFBCs. In both conditions, the associated multiplicities of the proposed code are significantly smaller than those of $[3,6]$. With 4-QAM signalling, at $\mathrm{CER}=10^{-3}$, the performance of the proposed code is only slightly better than that of the code in [3], while is $3 \mathrm{~dB}$ better than that of the code in [6] (see Fig. 1).

\section{CONCLUSION}

In this paper, we propose new algebraic multiuser $2 \times 2$ SFBCs for frequency selective MIMO MACs. Using a UB approximation, we first present the code design criteria. Combining algebraic perfect STBC structures, we show how to design a family of multiuser SFBCs to satisfy the design criteria, yet without peak-to-average penalty. Within this family, we present a code design example for a two-user case. It is shown that the proposed multiuser SFBC for frequency selective fading MIMO MACs outperforms all previously known codes.

\section{ACKNOWLEDGMENT}

The authors would like to thank M.E. Gärtner and H. Bölcskei for their fruitful discussions. The work of E. Viterbo 
was supported by the STREP project No. IST-026905 (MASCOT) within the Sixth Framework Programme of the European Commission.

\section{REFERENCES}

[1] H. Bölcskei and A.J. Paulraj, "Space-frequency coded broadband OFDM systems," Proc. IEEE Wireless Commun. Networking Conf. (WCNC), Chicago, IL, pp. 1-6, Sept. 2006.

[2] A.J. Paulraj, R. Nabar, and D. Gore, "Introduction to space-time wireless communications" Cambridge, UK, Cambridge Press, 2003.

[3] M.E. Gärtner and H. Bölcskei, "Multiuser space-time/frequency code design," Proc. IEEE Int. Symposium on Information Theory (ISIT), Seattle, WA, pp. 2819-2823, July 2006.

[4] F. Oggier, G. Rekaya, J.-C. Belfiore, and E. Viterbo, "Perfect space-time block codes," IEEE Trans. Inform. Theory, vol. 52, n. 9, pp. 3885-3902, September 2006.

[5] E. Biglieri, Y. Hong, E. Viterbo, "On fast-decodable space-time block codes," submitted to IEEE Trans. Inform. Theory, available in arxiv: CS.IT. 0708.2804.

[6] W. Zhang and K. Ben Letaief, "A systematic design of multiuser space-frequency codes for MIMO-OFDM systems," in Proc. IEEE International Conference on Communications (ICC'07), pp. 1054-1058, July 2007.

[7] E. Bayer-Fluckiger, F. Oggier, E. Viterbo, "Algebraic Lattice Constellations: bounds on performance," IEEE Transactions on Information Theory, vol. 52, n. 1, pp. 319-327, Jan. 2006.

[8] F. Oggier, E. Viterbo, "Algebraic number theory and code design for Rayleigh fading channels," in Foundations and Trends in Communications and Information Theory, vol. 1, pp. 333-415, 2004.

[9] J. Boutros and E. Viterbo, "Signal Space Diversity: a power and bandwidth efficient diversity technique for the Rayleigh fading channel," IEEE Transactions on Information Theory, vol. 44, n. 4, pp. 1453-1467, July 1998.

[10] M. Badr and J.-C. Belfiore, "Optimal Space-Time Codes for the noncooperative MAC channel," Asilomar Conference on Signals, Systems, and Computers, Nov. 2007 